\title{
Anästhesie und Outcome: Einfluss bei Kindern
}

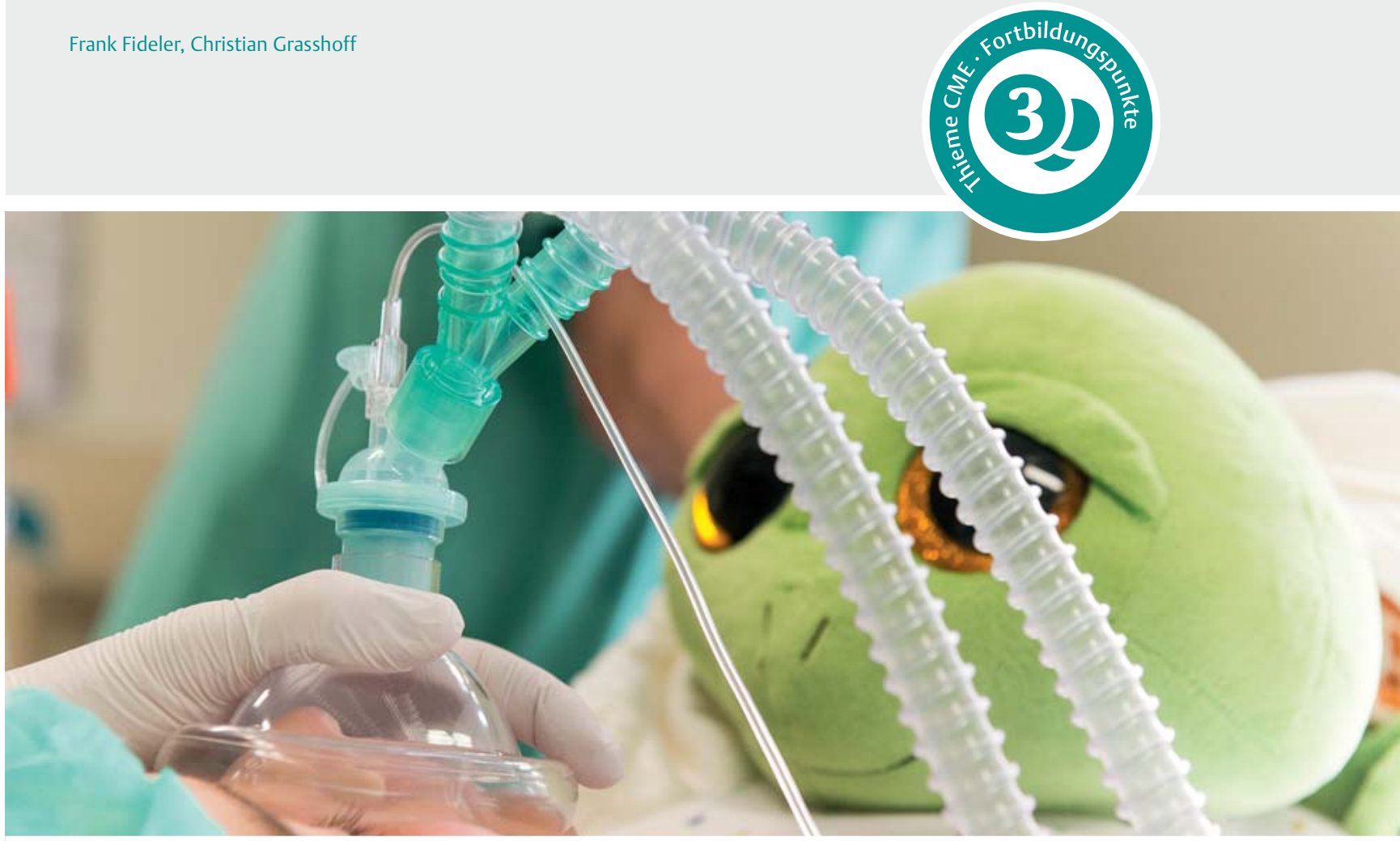

Quelle: KH Krauskopf.

Die operative Versorgung von Kindern birgt ein erhöhtes Risiko schwerer perioperativer Komplikationen und stellt hohe Anforderungen an die beteiligten Berufsgruppen. Um das perioperative Outcome von Kindern vonseiten der Anästhesie positiv zu beeinflussen, sind neben der Optimierung institutioneller Faktoren individuelle Möglichkeiten innerhalb der intraoperativen Phase der Narkoseeinleitung und -aufrechterhaltung bedeutend, insbesondere die Einstellung des adäquaten Blutdrucks.

\section{Einleitung}

Die operative Versorgung von Kindern stellt hohe Anforderungen an die beteiligten Berufsgruppen und gilt zu Recht als anspruchsvoll. Das Risiko schwerer perioperativer Komplikationen liegt nach aktuellen Daten der APRICOT-Studie bei 5,2\%. Als Risikofaktoren perioperativer Komplikationen zeigten sich hier hauptsächlich das Alter des Kindes, Grund- und Begleiterkrankungen sowie die körperliche Verfassung [1].

Da Anästhesisten weder diese Faktoren noch die benötigte Operation wesentlich beeinflussen können, ist es gerechtfertigt zu fragen, was der Anästhesist aktiv tun kann, um das perioperative Outcome von Kindern positiv zu beeinflussen. Die Möglichkeiten lassen sich grob in individuell und institutionell klassifizieren. Beide Gruppen der Einflussnahme sind wichtig und müssen unabhängig voneinander gestärkt werden. Naturgemäß war in den letzten Jahren der Fokus zahlreicher Publikationen besonders auf eine Verbesserung der individuellen Fähigkeiten gerichtet.

Zu den individuellen Möglichkeiten gehört u. a. die intraoperative Phase der Narkoseeinleitung und -aufrechterhaltung. Als wichtige Erkenntnis ist hier in den letzten Jahren immer mehr eine Aufrechterhaltung der Homöostase identifiziert worden [2]. Grob vereinfacht ist hierunter eine Vermeidung von arterieller Hypotension, Hyper-/ 


\section{präoperativ}

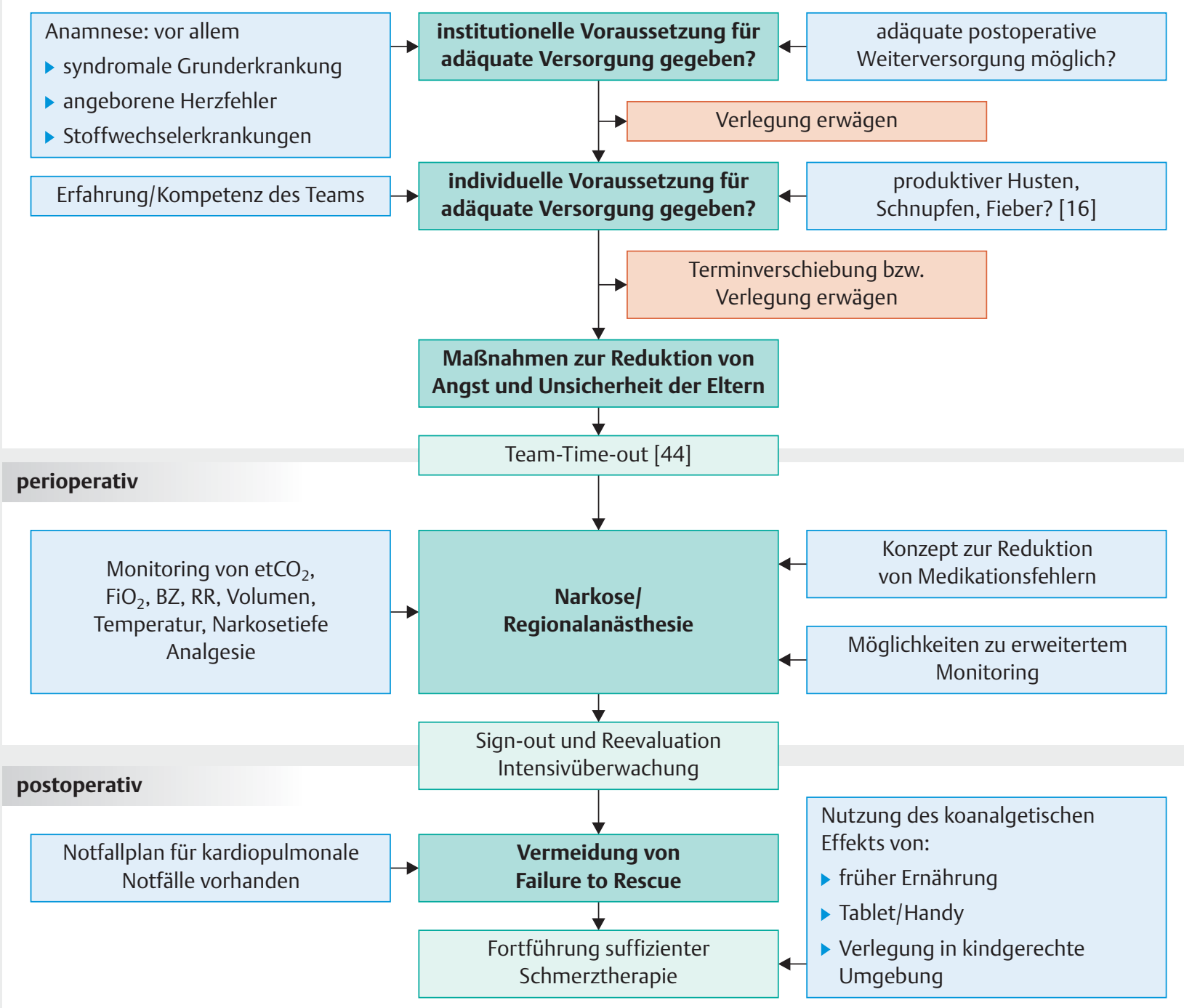

- Abb. 1 Algorithmus zur Evaluation Outcome-relevanter Entscheidungen.

Hypoglykämie, Hypoxämie, Hypokapnie und Hyponatriämie als Folge einer inadäquaten Flüssigkeitssubstitution zu verstehen. In diesem Artikel möchten wir uns aus diesem Themenbereich auf die Frage nach dem „richtigen“ Blutdruck beschränken, da hierzu zahlreiche neue Studien erschienen sind, die sich der Frage annehmen.

In der Grauzone zwischen individueller und institutioneller Entscheidung mit potenziellem Einfluss auf das Outcome haben wir die Frage einer möglichen Neurotoxizität diskutiert. Diese ist seit einiger Zeit nicht nur fachlich, sondern auch politisch und emotional im Fokus und wird in der Öffentlichkeit wahrgenommen [3].
Genauso wichtig ist allerdings die Optimierung der institutionellen Versorgung mit potenzieller Verbesserung des Outcomes. Dieser Bereich wird schnell übersehen, hält jedoch zahlreiche Möglichkeiten der Einflussnahme bereit.

Die wichtigsten Punkte zum Thema individuelle und institutionelle Möglichkeiten der Verbesserung des Outcomes sind in $>$ Abb. 1 zusammengefasst, die sowohl die präoperative, als auch die intra- und postoperative Phase beinhaltet. 


\section{Institutionelle Ansätze - Was können wir für Eltern und Kinder tun?}

Antizipiert man die Angst der Eltern vor unerwünschten Folgen des Eingriffs und der Anästhesie, so erscheint diese zumindest für die ganz kleinen Patienten berechtigt, wenn man die perioperative Mortalität betrachtet. In einem Review-Artikel haben McCann und Schouten hierzu ausgeführt, dass sie die anästhesiebedingte Mortalität mit 0,65-0,98 Fällen/10000 Narkosen als gering einstufen, während die perioperative Mortalität besonders bei Neonaten immer noch sehr hoch ist [2]. So ist die perioperative Sterblichkeit für Neugeborene in den USA ungefähr 70-mal höher als für Kinder, die älter als 10 Jahre sind [4]. Die höhere Sterblichkeit begründet sich vor allem in der Art der Grunderkrankung und dem daraus resultierenden Grad der Komplexität des chirurgischen Eingriffs. Hiermit sind vor allem Kinder mit angeborenen Herzfehlern gemeint, die eine operative Korrektur desselben benötigen [4].

Jenseits der fachlichen Kriterien stellt eine hohe emotionale Belastung der Eltern eine nicht zu unterschätzende Herausforderung dar. So konnte Litman et al. in einer Untersuchung an gesunden Kindern im Bereich der ambulanten Chirurgie bereits 1996 zeigen, dass Mütter von Säuglingen offenbar besonders viel Angst vor der perioperativen Phase haben [5]. Da sich Angst und Unsicherheit der Eltern auf die Kinder übertragen [6], ist es gerechtfertigt zu fragen, inwiefern sich die emotionale Belastung der Kinder auf den postoperativen Gesundheitszustand auswirken kann. Kain et al. untersuchten hierzu 241 Kinder im Alter von 5 bis 12 Jahren, die sich einer Operation im HNO-Bereich unterziehen mussten. Sie beschrieben, dass ängstliche Kinder eine deutlich höhere Schmerzempfindlichkeit unmittelbar postoperativ als auch während der ersten 3 Tage zu Hause angaben. Der Schmerzmittelverbrauch war ebenfalls signifikant erhöht. Weiterhin traten bei diesen Kindern öfter ein Emergence Delirium und Schlafstörungen auf [7].

Ein aktuelles Thema ist in diesem Zusammenhang die Frage, inwieweit Eltern bei der medizinischen Versorgung ihrer Kinder anwesend sein sollen, um ihnen die Möglichkeit zu geben, selbige zu begleiten. Hierzu haben Sanders et al. eine bemerkenswerte prospektive Kohortenstudie durchgeführt, in der sie auf 22 pädiatrischen Intensivstationen untersuchten, ob die Anwesenheit der Eltern während eines Intubationsvorgangs die Qualität der durchgeführten Maßnahme beeinflusste [8]. Die Autoren konnten zeigen, dass die Anwesenheit von Familienmitgliedern nicht die Wahrscheinlichkeit eines erfolgreichen ersten Intubationsversuchs beeinflusste, nicht mit einer erhöhten Anzahl an Entsättigungsepisoden assoziiert war und nicht mit einem erhöhten Stresslevel des Teams einherging. Auch wenn wir Zweifel haben, ob wir diese
Praxis in unseren täglichen OP-Ablauf integrieren möchten, so zeigt die Studie doch sehr gut, dass wir die Familienangehörigen zunehmend in die Versorgung integrieren dürfen. Es ist völlig klar, dass das Ausmaß der Integration von den jeweiligen Bedingungen vor Ort abhängen wird.

\section{Merke}

Ängstliche Kinder haben postoperativ während der ersten Tage eine deutlich höhere Schmerzempfindlichkeit. Auch treten ein Emergence Delirium und Schlafstörungen bei ängstlichen Kindern postoperativ häufiger auf.

Es stellt sich nun die Frage, ob eine Verhaltensänderung bei Eltern und medizinischem Personal das postoperative Ergebnis beeinflussen kann. Zum ersten Punkt konnte die Arbeitsgruppe um Kain et al. zeigen, dass eine gezielte verhaltensorientierte präoperative Vorbereitung von Eltern und Kindern die Angst während der Narkoseeinleitung ähnlich effektiv reduzierte wie eine medikamentöse Prämedikation mit Midazolam. Weiterhin benötigten diese Kinder weniger postoperative Analgetika, konnten schneller aus dem Aufwachraum entlassen werden und zeigten weniger Anpassungsstörungen nach dem Eingriff [9].

Dieselbe Gruppe hat die Strategie einer Einflussnahme über eine Verhaltensänderung konsequent weiterentwickelt und die Auswirkungen einer Schulung des medizinischen Personals auf das postoperative Ergebnis untersucht. Die Autoren beschrieben, dass eine gezielte verhaltenstherapeutische Intervention beim medizinischen Personal zu einer Verbesserung der Häufigkeit von „erwünschtem“ Verhalten (ablenkende Gespräche, Humor, positive Umdeutung unangenehmer medizinischer Prozeduren) gegenüber den Kindern führte und darüber hinaus auch das Verhalten der Eltern dahingehend beeinflusste, dass diese häufiger ein „erwünschtes“ Verhalten zeigten, obwohl diese nicht verhaltenstherapeutisch geschult wurden [10]. Offenbar lässt sich bereits durch eine Schulung des Personals indirekt das Verhalten der Eltern gegenüber den Kindern positiv beeinflussen und damit das postoperative Ergebnis verbessern.

\footnotetext{
Merke

Eine gezielte verhaltensorientierte präoperative Vorbereitung von Eltern und Kindern kann die Angst während der Narkoseeinleitung ähnlich effektiv reduzieren wie eine Prämedikation mit Midazolam.
} 


\section{Individuelle Fertigkeiten - Bedeutung des Fallaufkommens und des Ausbildungsstands}

Im Zusammenhang mit der Indikation für einen operativen Eingriff muss auch entschieden werden, an welcher Institution der Eingriff stattfinden soll. Diese Entscheidung wird oft nach den chirurgischen Kapazitäten getroffen, ist aber auch mit den anästhesiologischen Möglichkeiten verknüpft. Betrachtet man in diesem Zusammenhang das Fallaufkommen einer Institution, so sind beide Aspekte nur schwer voneinander zu trennen.

In einer Studie haben Mudumbai et al. über einen Zeitraum von 10 Jahren die Häufigkeit von kinderanästhesiologischen Eingriffen in Kalifornien nachuntersucht. Es stellte sich heraus, dass an fast $90 \%$ der Krankenhäuser auch komplexe Eingriffe vorgenommen wurden, obwohl die Fallzahlen unter 100 per anno lagen [11]. Hierdurch ist noch keine Aussage bezüglich der Qualität der Versorgung getroffen. Zieht man jedoch zur Beurteilung Daten hinzu, die ganz allgemein das Outcome von Patienten an Krankenhäusern mit geringen Fallzahlen mit dem an Häusern mit großen Fallzahlen vergleichen, so erzielten die größeren Häuser bessere Endergebnisse [12,13]. Die Autoren analysierten hierzu die Daten von ca. 85000 Patienten, die sich in einem Zeitraum von 2005 bis 2007 einem allgemein- oder gefäßchirurgischen Eingriff unterzogen. Sie unterteilten die beteiligten Krankenhäuser anhand der risikoadjustierten Mortalität in Quintilen und bestimmten die Häufigkeit von schweren Komplikationen in den jeweiligen Gruppen sowie das Risiko, an diesen zu versterben. Ghaferi et al. fanden heraus, dass sich die Mortalität hierbei um mehr als den Faktor 3 zugunsten der Krankenhäuser mit großem Fallaufkommen unterschied [13]. Die Gründe hierfür sind vor allem in den unterschiedlichen Möglichkeiten zu suchen, entstehende Komplikationen zu beherrschen. So kam es nach dem Auftreten von schweren Komplikationen in Krankenhäusern mit geringem Fallaufkommen 2- bis 3-mal häufiger zum Versterben der betroffenen Patienten, dem sogenannten „Failure to Rescue“ [12,13].

Für die Versorgung von Kindern existieren keine derartigen Untersuchungen, nicht zuletzt, weil derartige Fallzahlen kaum zu erreichen sind. Wir gehen jedoch davon aus, dass die für Erwachsene bezüglich des Umgangs mit schweren Komplikationen gefundenen Ergebnisse auch für ein pädiatrisches Patientenkollektiv zutreffen.

\footnotetext{
Merke

„Failure to Rescue“ bezeichnet das Versterben von Patienten nach postoperativen Komplikationen und tritt bei Erwachsenen in Krankenhäusern mit geringem Fallaufkommen 2- bis 3-mal häufiger auf als in Krankenhäusern mit hohem Fallaufkommen.
}

Eine bessere Datenlage existiert bezüglich des Einflusses des individuellen Ausbildungsstands auf das Outcome. So wurde bereits 1991 publiziert, dass es einen Zusammenhang zwischen der Häufigkeit eines intraoperativen Herzstillstands bei Säuglingen und dem Ausbildungsstand des Anästhesisten gibt. Keenan et al. werteten hierzu über einen Zeitraum von 7 Jahren an einem großen universitären Zentrum gewonnene Daten retrospektiv aus und fanden heraus, dass es in der Gruppe der Säuglinge, die von nicht spezialisierten Anästhesisten versorgt wurden, zu mehr intraoperativen Herzstillständen kam als in der Vergleichsgruppe [14]. Unterstützt wurden die Ergebnisse der Studie durch Auroy et al., die beschrieben, dass sich eine 5-fache Reduktion der schweren Komplikationen durch den Einsatz erfahrener Anästhesisten erzielen lässt. Als erfahren gelten hier Anästhesisten, die mehr als 200 Kinder im Jahr versorgten, gegenüber den unerfahrenen Anästhesisten, die weniger als 100 Fälle betreuten [15].

Die bedeutendste Studie zu diesem Thema wurde 2010 publiziert. Die Autoren untersuchten in einer prospektiven Kohortenstudie den Zusammenhang zwischen dem perioperativen Auftreten respiratorischer Komplikationen in Abhängigkeit von der familiären Vorgeschichte und der anästhesiologischen Versorgung [16]. Bezüglich des anästhesiologischen Managements arbeitete die Gruppe heraus, dass es bei einer intravenösen Narkoseeinleitung im Gegensatz zu einer inhalativen Einleitung zu weniger respiratorischen Komplikationen kam. Umgekehrt verhielt es sich bei der Fortführung der Anästhesie. Bezüglich des Einflusses des Ausbildungsstands der Anästhesisten war die Betreuung durch spezialisierte Kinderanästhesisten von signifikantem Vorteil bei der Vermeidung von Atemwegskomplikationen. Bestätigt wird dies durch die prospektiv erhobenen Ergebnisse der multizentrischen Kohortenstudie APRICOT. Die Bewertung von 31127 Anästhesien hinsichtlich der Häufigkeit schwerer Komplikationen ergab, dass die Erfahrung des dienstältesten Anästhesisten, gemessen in Jahren, die Komplikationshäufigkeit senkt [1].

Ein weiterer wichtiger Punkt zur Verbesserung der Anästhesiequalität liegt in der Vermeidung von Dosierungsfehlern. Für Kinder wissen wir, dass die Probleme hauptsächlich bei Ampullenverwechslungen, falsch aufgezogenen Spritzen und Dosierungsfehlern liegen. Eindrückliche Ergebnisse zeigte eine prospektive Beobachtungsstudie an 277 Patienten mit 3671 perioperativen Medikamentenapplikationen. Bei jedem 20. Patienten (5,3\%) kam es hierbei zu Medikationsfehlern respektive unerwünschten Medikamentenwirkungen. Diese Zahlen liegen wesentlich höher als bislang aus retrospektiven Studien angenommen [17]. Ein relevanter Faktor zur Risikoreduktion liegt demnach im reibungslosen Zusammenspiel institutioneller Voraussetzungen und individueller Erfahrung [18-20]. 
Aus den hier genannten Studien lässt sich schlussfolgern, dass jede Institution sorgfältig prüfen sollte, ob ihre (institutionelle) Erfahrung und der Ausbildungsstand der Mitarbeiter eine komplikationsarme Versorgung besonders der Säuglinge und Neonaten sicherstellen können. Bei großen und seltenen Operationen in diesem Patientengut ist die Versorgung an einem hierfür spezialisierten Zentrum anzustreben.

Merke

Die Häufigkeit von Medikationsfehlern, respiratorischen Komplikationen und intraoperativen Herzstillständen lässt sich durch institutionelle Maßnahmen sowie individuelle Schulung und Erfahrung reduzieren.

\section{Individuelles Management - intraoperative Kontrolle des Blutdrucks}

Die intraoperative Kontrolle des Blutdrucks wurde zuletzt bezüglich ihrer Wertigkeit für den Gesundheitszustand der operierten Kinder vielfach diskutiert [2]. So ist der Blutdruck zwar kein Surrogatparameter für den Blutfluss, aber wichtig für die Autoregulation der Gewebeperfusion. In diesem Zusammenhang ergaben sich folgende Fragen:

- Wie ist der Blutdruck zu messen?

- Beeinflusst der Blutdruck den Gesundheitszustand der Kinder und, wenn ja, wie?

- Wie ist ein altersbezogener adäquater Blutdruck zu definieren?

\section{Wie ist der Blutdruck zu messen?}

Entscheidend für die Messung valider Blutdruckwerte ist, dass der tatsächliche Blutdruck umso mehr überschätzt wird, je kleiner das Kind und je niedriger der Blutdruck ist. Grundvoraussetzungen für eine verlässliche Messung sind deshalb immer die für den Patienten richtige Manschettengröße (zu schmale Manschetten führen zu falsch hohen Werten) sowie die an vielen Blutdruckmessgeräten notwendige richtige Alterseinstellung [21].

\section{Beeinflusst der Blutdruck den Gesundheits- zustand der Kinder und, wenn ja, wie?}

Es ist zu beurteilen, ob ein niedriger intraoperativer Blutdruck den Gesundheitszustand beeinflusst. Im Jahr 2014 haben McCann et al. eine Fallserie von 6 kinderchirurgischen Patienten publiziert, die postoperativ an einer neu aufgetretenen Enzephalopathie mit Krampfanfällen und teilweise schweren Entwicklungsverzögerungen litten. Eines der Kinder verstarb an den Folgen der Enzephalopathie. In der retrospektiven Analyse der intraoperativen Vitalparameter wurden verschiedene Parameter als ursächlich für die zerebralen Veränderungen angesehen, u.a. das Auftreten einer intraoperativen Hypoglykämie, Hypothermie, Hyperoxie und Hypokapnie. Als besonders relevant für das Entstehen einer postoperativen Enzephalopathie bewerteten die Autoren jedoch das Absinken des systolischen Blutdrucks unter $60 \mathrm{mmHg}$ in der Mehrzahl der gemessenen Werte, obwohl bei diesen Patienten nur ca. $11 \%$ der Blutdruckwerte mehr als eine Standardabweichung unterhalb des Mittelwerts der offiziellen Definition einer Hypotension $(45 \mathrm{mmHg})$ der Gesellschaft für Pediatric Anesthesia (2009) lagen [22].

Die Relevanz der Aufrechterhaltung eines adäquaten Blutdrucks für die Wahrung der zerebralen Perfusion wird durch neuere Untersuchungen von Michelet et al. unterstützt. In einer prospektiven Beobachtungsstudie untersuchten die Autoren die Effekte intraoperativer Blutdruckänderungen bei Säuglingen, die jünger als 3 Monate waren, auf die zerebrale Sauerstoffsättigung, die mittels Nah-Infrarot-Spektroskopie gemessen wurde. Sie berichteten, dass ein Abfall des systolischen Blutdrucks um mehr als 20\% des Ausgangswerts mit einer mehr als 10-prozentigen Möglichkeit verbunden war, dass es zu einer zerebralen Entsättigung kam [23].

\section{Wie ist ein altersbezogener adäquater Blutdruck zu definieren?}

Mit der Bewertung der genannten Studie ist die Frage verbunden, wie ein altersbezogener adäquater Blutdruck zu definieren ist. Bis vor Kurzem war völlig unklar, in welchem Bereich Normwerte für den Blutdruck bei Kindern in der perioperativen Phase zu definieren sind. Durch die Studie von de Graaff et al. verfügen wir erstmals über Referenzwerte, die den tatsächlichen intraoperativen Verlauf eines Patientenkollektivs widerspiegeln. Hierzu untersuchten die Autoren in einer retrospektiven Kohortenstudie 116362 Kinder unter 18 Jahren. Dabei lagen sowohl die Werte der 50. Perzentile als auch diejenigen innerhalb der 2-fachen Standardabweichung deutlich niedriger als die der wachen Patienten. Eingeschlossen wurden lediglich Patienten mit ASA I und II. Bedingt durch das Design der Arbeit waren keine Aussagen darüber möglich, ob diese Blutdruckwerte als „ausreichend“ gelten können, da Fragen zur Organperfusion mit Enzephalopathie oder Nierenversagen ebenso wie zur Länge des Krankenhausaufenthalts oder zur Mortalität unbeantwortet blieben [24]. Gerade bei Patienten mit Vorerkrankungen ist jedoch anzunehmen, dass die Autoregulationskurve hin zu höheren Blutdruckwerten verschoben ist [25].

Eine Umfrage unter Mitgliedern der Society of Pediatric Anesthesia (SPA) und der Association of Paediatric Anaesthetists (APA) 2009 zur Definition intraoperativer Hypotension zeigte zum Teil beträchtliche Unterschiede zwischen den Werten, die von den 483 teilnehmenden Kinderanästhesisten als behandlungsbedürftig angesehen wurden. Für 2-jährige Kinder beispielsweise wurden systolische Werte von $55 \mathrm{mmHg}$ (SPA) bis $60 \mathrm{mmHg}$ (APA) als Schwellenwerte einer intraoperativen Hypotension angegeben [26]. Legt man diese Definition zugrunde, so 
zeigen 2 neuere retrospektive Studien, dass bei einer relevanten Anzahl der Kinder perioperativ Blutdrücke gemessen werden, die als kritisch anzusehen sind. Abhängig vom Alter wurden bis zu $21 \%$ der Patienten mit einem mittleren Blutdruck von weniger als $35 \mathrm{mmHg}$ gefunden, nach der Einleitung von Säuglingen sogar 25,5\% des untersuchten Kollektivs [27, 28].

Zusammenfassend lässt sich sagen, dass die Evidenz für einzuhaltende untere Grenzen des Blutdrucks schwach ist. Daher wird von einigen Autoren ein „adäquater Blutdruck“ als derjenige bezeichnet, bei dem die Organperfusion, vor allem aber der zerebrale Blutfluss (cerebral blood flow, CBF), im Normbereich liegen. Der CBF ist dabei direkt vom Blutdruck abhängig.

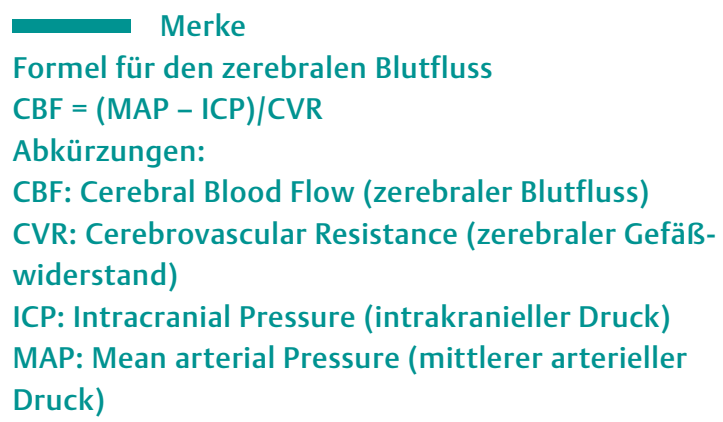

Der CBF ist das Blutvolumen, das pro Zeiteinheit ein Hirnareal durchströmt und die Sauerstoffversorgung der Neurone gewährleistet. Er ist zudem abhängig von den Stellgrößen, die den zerebrovaskulären Gefäßwiderstand beeinflussen. Dazu gehören als wichtige Parameter der $\mathrm{pH}, \mathrm{pCO}_{2}, \mathrm{pO}_{2}$, aber auch Änderungen der Kalium- oder Adenosinkonzentration. Unter physiologischen Bedingungen besteht für den CBF dabei eine zerebrale Autoregulation. Bei gleichbleibendem zerebralem Perfusionsdruck kann das Gehirn durch Veränderung des zerebrovaskulären Gefäßwiderstands der Arteriolen bei Schwankungen des MAP den CBF konstant halten (Bayliss-Effekt) $[2,25]$.

Eine Quantifizierung des zerebralen Blutflusses ist naturgemäß schwierig, als Surrogatparameter bietet sich die zerebrale Sauerstoffsättigung an, die mittels NIRS-Verfahren gemessen werden kann. So konnten Rhondali et al. in einer retrospektiven Studie an 338 Kindern unter 6 Monaten zeigen, dass sich die zerebrale Perfusion anhand des mittleren arteriellen Blutdrucks gut abschätzen lässt. Die Autoren empfehlen für Patienten in dieser Altersklasse, dass ein mittlerer arterieller Blutdruck von mehr als $35 \mathrm{mmHg}$ nicht unterschritten werden soll, um kritische Verringerungen des zerebralen Blutflusses zu vermeiden [29]. Dies korreliert auch mit den von de Graaff erhobenen Werten [24]. Für Säuglinge über 6 Monate sollte ein Grenzwert des mittleren arteriellen Druckes von $43 \mathrm{mmHg}$ nicht unterschritten werden
- Tab. 1 Empfohlene untere Grenzwerte für den mittleren arteriellen Blutdruck von Säuglingen.

\begin{tabular}{|l|l|}
\hline Alter & unterer Grenzwert \\
\hline$<6$ Monaten & $35 \mathrm{mmHg}$ \\
\hline$>6$ Monate & $43 \mathrm{mmHg}$ \\
\hline
\end{tabular}

$\left(\triangleright\right.$ Tab. 1), insbesondere nicht in Verbindung mit $\mathrm{PaCO}_{2}-$ und Hb-Abfällen [30].

Der Einfluss einer Hypokapnie während einer SevofluranAnästhesie auf Perfusion und Metabolismus im sich entwickelnden Gehirn wurde tierexperimentell an 28 Ferkeln belegt. Insbesondere die Kombination aus Hypotension und Hypokapnie führte zu erkennbaren Veränderungen der zerebralen Perfusion, Zeichen neuronaler Dysfunktion und früher neuronaler Ischämie [31].

\section{Merke}

Die Vermeidung intraoperativer Hypotensionen und Hypokapnien ist wichtiger Bestandteil der perioperativen Homöostase und Voraussetzung zur Aufrechterhaltung der zerebralen Perfusion. Insbesondere die Kombination von Hypotension und Hypokapnie gilt es zur Vorbeuge neuronaler Ischämien zu vermeiden.

\section{Einfluss von Anästhetika auf das sich entwickelnde Gehirn}

In den letzten Jahren hat die Frage nach einer potenziellen Neurotoxizität von Allgemeinanästhetika die Diskussion bezüglich des Outcomes relevanter Parameter im Bereich der Kinderanästhesie dominiert. So befassten sich allein im Jahr 2011 nahezu die Hälfte aller Publikationen im Bereich der Kinderanästhesie mit dem Thema der möglichen negativen Einflüsse von Anästhetika auf das sich in Entwicklung befindliche Gehirn [32]. Die in Tierversuchen gewonnenen Ergebnisse und auch die Resultate von In-vitro-Experimenten zeigten übereinstimmend, dass alle untersuchten Allgemeinanästhetika unabhängig vom molekularen Wirkmechanismus zu neuroanatomischen Veränderungen führten. Die neuroanatomischen Veränderungen wiederum induzierten lang anhaltende funktionelle Defizite in allen untersuchten Spezies bis hin zu Primaten [33].

Im Gegensatz zu den experimentellen Daten waren die aus kontrollierten klinischen Studien erzielten Ergebnisse weit weniger beunruhigend. So zeigten sowohl die gerade erst publizierte Zwischenanalyse der GAS-Studie („General Anesthesia versus Spinal Anesthesia“) als auch der PANDA-Studie („Pediatric Anesthesia and Neurodevelopment Assessment“), dass eine kurze, einmalige Ex- 
position gegenüber Anästhetika nicht zu Verschlechterungen in der neurokognitiven Entwicklung der untersuchten Kinder führte [34, 35].

Diese Schwierigkeiten der inhaltlichen Zusammenführung der diskrepanten Ergebnisse der Laboruntersuchungen und klinischen Studien sind sicherlich zum einen in der komplexen Konstellation zwischen frühkindlicher Applikation von Anästhetika und einer um viele Jahre verzögerten klinischen Manifestation zu finden: Die Phase intensiver Synapsenneubildung, der „Brain Growth Spurt“, erfolgt beim Menschen mit großen Unterschieden zu den meisten Vergleichsspezies, vor allem aber auch mit individueller und regionaler Varianz. In dieser Phase gelten Neurone als besonders empfindlich gegenüber äußeren und inneren Einflüssen. Beim Menschen dauert diese Phase deutlich länger als bei Nagern, Schweinen und Affen [36]. Weiterhin sind beim Menschen nicht alle Hirnregionen gleichzeitig betroffen, und es zeigen sich auch innerhalb einer Hirnregion deutliche zeitliche Unterschiede bezüglich des „Brain Growth Spurts“, wie in Untersuchungen an verschiedenen kortikalen Schichten gezeigt werden konnte [37].

Es ist kaum möglich, einzelne Hirnregionen funktionell spezifischen Verhaltenstests zu unterziehen. Die weitere Entwicklung der Kinder mit einem Aufwachsen in einem komplexen, stimulierenden sozialen Umfeld erschwert das spätere Herstellen eines monokausalen Zusammenhangs ebenfalls. Weiterhin verfügt das menschliche Gehirn über zahlreiche Kompensationsmechanismen, um eventuell entstandene Beeinträchtigungen auszugleichen. So untersuchte eine große schwedische Kohortenstudie deshalb bei allen Kindern der Geburtsjahrgänge 1973-1993 Faktoren, die nach OP/Narkose die Ergebnisse des Schulabschlusses sowie den IQ-Test bei der Einberufung zum Militär beeinflussten. Eine einzelne Operation in Vollnarkose im Alter von unter 4 Jahren zeigte dabei deutlich geringere Auswirkungen auf die durchschnittliche Schulnote als die Bildung der Mutter, das Geschlecht des Kindes oder der Geburtszeitpunkt innerhalb eines Kalenderjahrs [38].

Ausgehend von dieser Datenlage veröffentlichte die FDA am 14. Dezember 2016 jedoch einen Sicherheitshinweis und ließ die Etiketten gebräuchlicher Medikamente zur Anästhesie und Sedierung bei Kindern unter 3 Jahren und Schwangeren im 3. Trimenon mit einem Warnhinweis versehen [39].

Gänzlich fehlen bislang allerdings Daten, die speziell das Risiko einer Anästhesieexposition des Feten in utero auf dessen weitere neurologische Entwicklung bewerten [40]. Ebenso ist das Verschieben oder Verhindern einer klar indizierten Operation oder Diagnostik/Intervention in Allgemeinanästhesie bei Kindern unter 3 Jahren auf- grund der vorliegenden wissenschaftlichen Evidenz nicht zu rechtfertigen [41].

Auch wenn dieser Warnhinweis nur im Zuständigkeitsbereich der FDA gültig ist, gehen wir davon aus, dass er zu einer zunehmenden Verunsicherung nicht nur der Eltern, sondern auch bei Anästhesisten in Deutschland führen wird. Es ist daher zu befürchten, dass es zu Verzögerungen bei notwendigen chirurgischen und diagnostischen Prozeduren kommt und der hierdurch verursachte Schaden den einer möglichen Belastung durch eine Anästhesie überwiegt.

\begin{abstract}
Merke
Bislang existiert keine ausreichende Evidenz, dass eine mutmaßlich anästhetikainduzierte Neurotoxizität beim Menschen klinisch relevant ist. Das Verzögern einer klar indizierten chirurgischen oder diagnostischen Prozedur in Allgemeinanästhesie bei Kindern ist deshalb nicht zu rechtfertigen.
\end{abstract}

\section{Wie sind die Perspektiven?}

Ziel der vorliegenden Arbeit war es, anhand ausgewählter Punkte institutionelle und individuelle anästhesiologische Möglichkeiten der Verbesserung des Outcomes von Kindern nach operativen Eingriffen darzustellen. Die Auswahl der hier aufgeführten Themen ist naturgemäß unvollständig und unterliegt einer subjektiven Einschätzung, uns waren hierbei 2 Dinge besonders wichtig: Zum einen eine Betonung der strukturellen oder institutionellen Voraussetzungen, die nicht zuletzt auch die individuellen Möglichkeiten der an einer Institution beschäftigten Anästhesisten bestimmen. Die andere wichtige Erkenntnis ergibt sich aus der begrenzten Aussagefähigkeit der zum Thema bereits publizierten Studien.

Wie sind also die Perspektiven? In den letzten Jahren sind Initiativen implementiert worden, welche die Verbesserung der Durchführung von Kinderanästhesien zum Ziel haben. Hierzu gehört die Safe tots.org Initiative (Safe Anesthesia For Every Tot Initiative), die zum einen die perioperativen Risikofaktoren bezüglich einer zerebralen Morbidität thematisiert, zum anderen aber auch den institutionellen Kontext einbezieht $[42,43]$.

Die deutlichen Unterschiede in der Häufigkeit für das Auftreten schwerer Komplikationen zwischen den teilnehmenden Ländern der APRICOT-Studie weisen ebenfalls daraufhin, dass institutionelle und individuelle Maßnahmen in der Zukunft Potenzial zur Qualitätsverbesserung bieten [1]. Es ist anzunehmen, dass bei einer gleichbleibenden Anzahl von Kinderanästhesien diese zunehmend in dafür ausgerichteten großen Zentren durchgeführt werden. 


\section{KERNAUSSAGEN}

- Eine verhaltensorientierte präoperative Vorbereitung von Eltern und Kindern wirkt sich positiv auf den postoperativen Verlauf der Kinder aus.

- Maßnahmen zur Reduktion von Angst und Unsicherheit der Eltern sind im postoperativen Verlauf verbunden mit geringerem Schmerzmittelbedarf, weniger Emergence Delir und weniger Schlafstörungen.

- Ein hohes Fallaufkommen und die Erfahrung des Anästhesisten wirken sich positiv aus hinsichtlich der Anzahl intraoperativer Herzstillstände, Vermeidung von Atemwegskomplikationen sowie der Häufigkeit postoperativer Komplikationen.

- Institutionelle Konzepte zur Reduktion von Medikationsfehlern beeinflussen das Behandlungsergebnis positiv.

- Zur Aufrechterhaltung einer ausreichenden zerebralen Perfusion sollen Hypotensionen vor allem in Verbindung mit $\mathrm{p}_{\mathrm{a}} \mathrm{CO}_{2}$ - und $\mathrm{Hb}$-Abfällen vermieden werden.

- Failure to Rescue“ ist abhängig vom Fallaufkommen der Klinik. Hohe Fallzahlen wirken sich positiv aus.

- Zwischenergebnisse von GAS- und PANDA-Studie zeigen, dass die einmalige kurze Exposition gegenüber Anästhetika nicht zu einer Verschlechterung der neurologischen Entwicklung führt.

- Das Verzögern einer klar indizierten chirurgischen oder diagnostischen Prozedur in Allgemeinanästhesie bei Kindern ist nach aktueller Datenlage nicht zu rechtfertigen

\section{Interessenkonflikt}

Die Autoren erklären, dass keine Interessenkonflikte vorliegen.

Über die Autoren

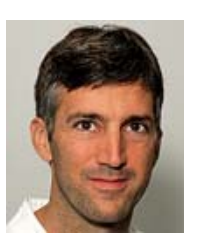

\section{Frank Fideler}

Dr. med., DESA, Oberarzt an der Klinik für Anästhesiologie und Intensivmedizin, Universitätsklinikum Tübingen. Klinische Schwerpunkte: Kinderanästhesie, Regionalanästhesie sowie ultraschallgesteuerte Verfahren (hier Tätigkeit als DEGUM-Kursleiter).

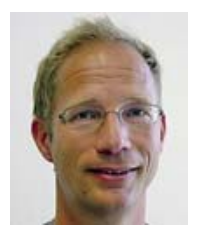

\section{Christian Grasshoff}

Prof. Dr. med., Stellvertretender Ärztlicher Direktor an der Klinik für Anästhesiologie und Intensivmedizin, Universitätsklinikum Tübingen. Klinische Schwerpunkte: Transplantations- und Kardioanästhesie sowie anästhesiologische Versorgung von Patienten mit großen Lebereingriffen. Schwerpunkt des wissenschaftlichen Interesses: molekulare Wirkmechanismen von Allgemeinanästhetika in neuronalen Netzwerken.

\section{Dr. med. Frank Fideler}

Klinik für Anästhesiologie und Intensivmedizin

Universitätsklinikum Tübingen

Hoppe-Seyler-Straße 3

72076 Tübingen

frank.fideler@med.uni-tuebingen.de

\section{Literatur}

[1] Habre W, Disma N, Virag K et al. Incidence of severe critical events in paediatric anaesthesia (APRICOT): a prospective multicentre observational study in 261 hospitals in Europe. Lancet Respir Med 2017; 5: 412-425

[2] McCann ME, Schouten AN. Beyond survival; influences of blood pressure, cerebral perfusion and anesthesia on neurodevelopment. Paediatr Anaesth 2014; 24: 68-73

[3] Jimenez F. Vollnarkose verschlechtert dauerhaft das Gedächtnis. Im Internet: www.welt.de/gesundheit/article128867496/ Vollnarkose-verschlechtert-dauerhaft-das-Gedaechtnis.html; Stand: 27.06.2017

[4] Flick RP, Sprung J, Harrison TE et al. Perioperative cardiac arrests in children between 1988 and 2005 at a tertiary referral center: a study of 92,881 patients. Anesthesiology 2007; 106 : 226-237

[5] Litman RS, Berger AA, Chhibber A. An evaluation of preoperative anxiety in a population of parents of infants and children undergoing ambulatory surgery. Paediatr Anaesth 1996; 6: 443-447

[6] Chorney JM, Tan ET, Kain ZN. Adult-child interactions in the postanesthesia care unit: behavior matters. Anesthesiology 2013; 118: 834-841

[7] Kain ZN, Mayes LC, Caldwell-Andrews AA et al. Preoperative anxiety, postoperative pain, and behavioral recovery in young children undergoing surgery. Pediatrics 2006; 118: 651-658

[8] Sanders RC jr., Nett ST, Davis KF et al. Family presence during pediatric tracheal intubations. JAMA Pediatr 2016; 170: e154627

[9] Kain ZN, Caldwell-Andrews AA, Mayes LC et al. Family-centered preparation for surgery improves perioperative outcomes in children: a randomized controlled trial. Anesthesiology 2007; 106: 65-74

[10] Martin SR, Chorney JM, Tan ET et al. Changing healthcare providers' behavior during pediatric inductions with an empirically based intervention. Anesthesiology 2011; 115: 18-27

[11] Mudumbai SC, Honkanen A, Chan J et al. Variations in inpatient pediatric anesthesia in California from 2000 to 2009: a caseload and geographic analysis. Paediatr Anaesth 2014; 24 : 1295-1301

[12] Ghaferi AA, Birkmeyer JD, Dimick JB. Variation in hospital mortality associated with inpatient surgery. N Engl J Med 2009; 361: 1368-1375

[13] Ghaferi AA, Birkmeyer JD, Dimick JB. Hospital volume and failure to rescue with high-risk surgery. Med Care 2011; 49: 1076-1081

[14] Keenan RL, Shapiro JH, Dawson K. Frequency of anesthetic cardiac arrests in infants: effect of pediatric anesthesiologists. J Clin Anesth 1991; 3: 433-437

[15] Auroy Y, Ecoffey C, Messiah A, et al. Relationship between complications of pediatric anesthesia and volume of pediatric anesthetics. Anesth Analg 1997; 84: 234-235 
[16] von Ungern-Sternberg BS, Boda K, Chambers NA et al. Risk assessment for respiratory complications in paediatric anaesthesia: a prospective cohort study. Lancet 2010; 376 (9743): 773783

[17] Nanji KC, Patel A, Shaik S et al. Evaluation of Perioperative Medication Errors and Adverse Drug Events. Anesthesiology 2016; 124: 25-34

[18] Merry AF, Anderson BJ. Medication errors - new approaches to prevention. Paediatr Anaesth 2011; 21: 743-753

[19] Llewellyn RL, Gordon PC, Wheatcroft D et al. Drug administration errors: a prospective survey from three South African teaching hospitals. Anaesth Intensive Care 2009; 37: 93-98

[20] Kozer E, Seto W, Verjee Z et al. Prospective observational study on the incidence of medication errors during simulated resuscitation in a paediatric emergency department. BMJ 2004; 329 (7478): 1321-1321

[21] Clark JA, Lieh-Lai MW, Samaik A, Mattoo TK. Discrepancies between direct and indirect blood pressure measurements using various recommendations for arm cuff selection. Pediatrics 2002; 110: 920-923

[22] McCann ME, Schouten AN, Dobija N et al. Infantile postoperative encephalopathy: perioperative factors as a cause for concern. Pediatrics 2014; 133: e751-e757

[23] Michelet D, Arslan O, Hilly J et al. Intraoperative changes in blood pressure associated with cerebral desaturation in infants. Paediatr Anaesth 2015; 25: 681-688

[24] de Graaff JC, Pasma W, van Buuren S et al. Reference Values for Noninvasive Blood Pressure in Children during Anesthesia: A Multicentered Retrospective Observational Cohort Study. Anesthesiology 2016; 125: 904-913

[25] Williams M, Lee JK. Intraoperative blood pressure and cerebral perfusion: strategies to clarify hemodynamic goals. Paediatr Anaesth 2014; 24: 657-667

[26] Nafiu OO, Voepel-Lewis T, Morris M et al. How do pediatric anesthesiologists define intraoperative hypotension? Paediatr Anaesth 2009; 19: 1048-1053

[27] Simpao AF, Ahumada LM, Gálvez JA et al. The timing and prevalence of intraoperative hypotension in infants undergoing laparoscopic pyloromyotomy at a tertiary pediatric hospital. Paediatr Anaesth 2017; 27: 66-76

[28] Weber F, Honing GH, Scoones GP. Arterial blood pressure in anesthetized neonates and infants: a retrospective analysis of 1091 cases. Paediatr Anaesth 2016; 26: 815-822

[29] Rhondali O, André C, Pouyau A et al. Sevoflurane anesthesia and brain perfusion. Paediatr Anaesth 2015; 25: 180-185

[30] Rhondali O, Juhel S, Mathews $S$ et al. Impact of sevoflurane anesthesia on brain oxygenation in children younger than 2 years. Paediatr Anaesth 2014; 24: 734-740
[31] Ringer SK, Ohlerth S, Carrera I et al. Effects of hypotension and/or hypocapnia during sevoflurane anesthesia on perfusion and metabolites in the developing brain of piglets-a blinded randomized study. Paediatr Anaesth 2016; 26: 909-918

[32] Davidson AJ. Neurotoxicity and the need for anesthesia in the newborn: does the emperor have no clothes? Anesthesiology 2012; 116: 507-509

[33] Sanders RD, Hassell J, Davidson AJ et al. Impact of anaesthetics and surgery on neurodevelopment: an update. $\mathrm{Br} J$ Anaesth 2013; 110 (Suppl. 1): i53-i72

[34] Davidson AJ, Disma N, de Graaff JC et al. Neurodevelopmental outcome at 2 years of age after general anaesthesia and awake-regional anaesthesia in infancy (GAS): an international multicentre, randomised controlled trial. Lancet 2016; 387 (10015): 239-250

[35] Sun LS, Li G, Miller TL et al. Association between a single general anesthesia exposure before age 36 months and neurocognitive outcomes in later childhood. JAMA 2016; 315: 23122320

[36] Dobbing J, Sands J. Comparative aspects of the brain growth spurt. Early Hum Dev 1979; 3: 79-83

[37] Huttenlocher PR, Dabholkar AS. Regional differences in synaptogenesis in human cerebral cortex. J Comp Neurol 1997; 387: 167-178

[38] Glatz P, Sandin RH, Pedersen NL et al. Association of anesthesia and surgery during childhood with long-term academic performance. JAMA Pediatr 2017; 171: e163470

[39] FDA Drug Safety Communication: FDA review results in new warnings about using general anesthetics and sedation drugs in young children and pregnant women. Im Internet: https:// wayback.archive-it.org/7993/20170111071047/http://www. fda.gov/Drugs/DrugSafety/ucm532356.htm; Stand: 27.06.2017

[40] Andropoulos DB, Greene MF. Anesthesia and Developing Brains - Implications of the FDA Warning. N Engl J Med 2017; 376: 905-907

[41] Becke K, Eich C, Höhne C et al. Kinderanästhesie: Was wirklich wichtig ist. Dtsch Arztebl International 2017; 114: 166-169

[42] Im Internet: www.safetots.org; Stand: 27.06.2017

[43] Weiss M, Vutskits L, Hansen TG, Engelhardt T. Safe Anesthesia For Every Tot - The SAFETOTS initiative. Curr Opin Anaesthesiol 2015; 28: 302-307

[44] Haynes AB, Weiser TG, Berry WR. A surgical safety checklist to reduce morbidity and mortality in a global population. $N$ Engl J Med 2009; 360: 491-499

Bibliografie

DOI https://doi.org/10.1055/s-0042-118829

Anästhesiol Intensivmed Notfallmed Schmerzther 2017; 52: 513-523 ๔ Georg Thieme Verlag KG Stuttgart · New York ISSN 0939-2661 


\section{Punkte sammeln auf CME.thieme.de}

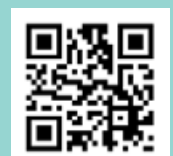

Diese Fortbildungseinheit ist 12 Monate online für die Teilnahme verfügbar.

Sollten Sie Fragen zur Online-Teilnahme haben, finden Sie unter cme.thieme.de/hilfe eine ausführliche Anleitung. Wir wünschen viel Erfolg beim Beantworten der Fragen!

Unter eref/thieme.de/ZZWHKY3 oder über den QR-Code kommen Sie direkt zum Artikel zur Eingabe der Antworten.

VNR 2760512017152373991

\section{Frage 1}

Was gehört nicht zur Aufrechterhaltung der perioperativen Homöostase im engeren Sinne?
A Normotension
B Normoglykämie
C Normomagnesiämie
D Normoxie
E Normonatriämie

\section{Frage 2}

Eine Reduktion von Angst und Unsicherheit bei den Eltern beeinflusst auch die kindlichen Patienten. Zu was führt diese Reduktion nicht?
A geringere Schmerzempfindlichkeit unmittelbar postoperativ
B seltenerem Erbrechen postoperativ
C geringerer Schmerzmittelverbrauch postoperativ
D selteneres Emergence Delir
E weniger Schlafstörungen

\section{Frage 3}

Was trifft für eine gezielte präoperative verhaltensorientierte Vorbereitung von Eltern und Kindern zu?

A Sie reduziert die Angst vor der Narkoseeinleitung ähnlich effektiv wie eine medikamentöse Prämedikation mit Midazolam.

B Sie zeigt keinen Einfluss auf den postoperativen Analgetikabedarf.

C Sie zeigt keine kürzere Aufenthaltsdauer im Aufwachraum.

D Sie reduziert den perioperativen Narkotikabedarf.

E Sie zeigt keinen Unterschied bezüglich Anpassungsstörungen nach dem Eingriff.

\section{Frage 4}

Was trifft für den Begriff „Failure to Rescue“ zu?

A Er bezeichnet die Möglichkeit, postoperativ entstehende Komplikationen zu beherrschen.

B Er verbessert die Chancen, eine postoperative Komplikation zu überleben.

C Er liegt in Krankenhäusern mit geringem Fallaufkommen 2bis 3-mal höher im Vergleich zu Krankenhäusern mit hohem Fallaufkommen.

D Er spielt bei Kindern keine Rolle.

E Er bezeichnet die Morbidität und Mortalität perioperativer Komplikationen.

\section{Frage 5}

Mit was ist ein fortgeschrittener individueller Ausbildungsstand nicht verbunden?
A mit weniger intraoperativen Herzstillständen
B mit häufigeren supraventrikulären Tachykardien
C mit weniger respiratorischen Komplikationen
D mit selteneren Medikationsfehlern
E mit weniger schwerwiegenden Komplikationen

\section{Frage 6}

Was trägt nicht zur Sicherheit der perioperativen Patientenversorgung bei?
A institutionelle Maßnahmen
$B$ individuelle Maßnahmen
C Team-Time-out
D Vermeidung von „Failure to Rescue“
E Anwesenheit der Eltern während der Einleitung

\section{Frage 7}

Welcher mittlere arterielle Blutdruck gilt für Säuglinge unter 6 Monaten als untere Grenze?
A $25 \mathrm{mmHg}$
B $30 \mathrm{mmHg}$
C $35 \mathrm{mmHg}$
D $40 \mathrm{mmHg}$
E $45 \mathrm{mmHg}$ 


\section{Punkte sammeln auf CME.thieme.de}

\section{Fortsetzung $\ldots$}

\section{Frage 8}

Welche Aussage zur Neurotoxizität von Anästhetika ist zutreffend?

A Neurotoxizität ist in Tierversuchen sicher nachgewiesen.

B Neurotoxizität hat zur Folge, dass bei Kindern unter 2 Jahren keine Narkose gemacht werden soll.

C GAS und PANDA-Studie zeigten neurokognitive Beeinträchtigungen der Patienten mit Vollnarkose.

D Neurotoxizität hat immer Lern- und Gedächtnisstörungen zur Folge.

E Neurotoxizität führt zu motorischer Entwicklungsverzögerung.

\section{Frage 9}

Durch was kann das Behandlungsergebnis nicht verbessert werden?

A Verlegung in geeignete Institution

B Vermeidung des Elternkontakts im Aufwachraum

C Einführung der WHO-Checkliste

D zusätzliche Sicherheitskontrollen bei der Medikamentengabe

E Strategien zur Vermeidung eines „Failure to Rescue“

\section{Frage 10}

Was gilt für den zerebralen Blutfluss nicht?

A Er ist definiert als $C B F=(M A P-I C P) / C V R$.

B Er ist abhängig vom Blutdruck des Patienten.

C Er ist ein wichtiger Parameter zur Vermeidung von Enzephalopathien.

D Er kann perioperativ direkt bestimmt werden.

E Er ist abhängig vom Gefäßwiderstand des Patienten. 